\title{
Local level statistics for optical and transport properties of disordered systems at finite temperature
}

\author{
A. Malyshev $* 1$ \\ ${ }^{1}$ GISC, Departamento de Física de Materiales, Universidad Complutense, Madrid, Spain
}

\begin{abstract}
It is argued that the (traditional) global level statistics which determines localization and coherent transport properties of disordered systems at zero temperature (e.g. the Anderson model)becomes inappropriate when it comes to incoherent transport. We define local level statistics which proves to be relevant for finite temperature incoherent transport and optics of one-dimensional systems (e.g. molecular aggregates, conjugated polymers, etc.).
\end{abstract}

PACS numbers: $78.30 . \mathrm{Ly}, 36.20 . \mathrm{Kd}, 72.20 . \mathrm{Ee}, 71.55 . J v$ 71.35.Aa

\section{INTRODUCTION}

Level statistics has extensively been studied in the connection with localization and transport properties of disordered systems (see e. g. Ref. [1] and references therein). In particular, nearest level spacing (NLS) distribution function $P(S)$ has a clear physical meaning and its relationship with localization properties is easily established: in the localized phase, energy levels are uncorrelated and can be infinitesimally close in energy (the corresponding wave functions can be localized far away from each other with a vanishing overlap), which gives rise to the Poisson NLS distribution. On the other hand, in the extended phase wave functions overlap well, which leads to strong level repulsion and results in the Wigner-Dyson NLS statistics (time-reversal symmetry is assumed hereafter). Various quantities associated with $P(S)$ were proposed as scaling variables to analyze metal-insulator transition point, critical exponents, mobility edges, and other characteristics relevant for coherent transport (see e. g. Ref. 2] and references therein). In the case of incoherent transport a quasi-particle can hop between different localized states because of interactions with environment (e.g. with a thermal bath as in the case of the phonon-assisted hopping). The rate (or probability) of hopping between two states is then proportional to the square of the matrix element of the interaction calculated between wavefunctions of the states. Hopping probability between states with vanishing overlap is therefore negligible (localized phase is considered hereafter); scattering rate is relatively large only for the states that overlap well. The latter introduces correlation between pairs of levels that are relevant for hopping. No such correlation is accounted for in the traditional global level statistics, we therefore define local level statistics that takes the correlation into account and proves to be adequate for incoherent transport in 1D disordered systems.

In the next section, we introduce a sample model of phonon-assisted hopping, section 3 presents a definition of the local NLS statistics in 1D, numerical results are discussed in section 4, while section 5 concludes the paper.

\section{DIFFUSION MODEL}

In this section we present an example model of quasi-particle diffusion in a random uncorrelated potential $\left\{\varepsilon_{n}\right\}$ mediated by weak coupling to a phonon system. The quasi-particle spectrum and wavefunctions $\left\{E_{\nu}, \Psi_{\nu}\right\}$ are defined as:

$$
\hat{H} \Psi_{\nu}=E_{\nu} \Psi_{\nu}, \quad \hat{H}=\sum_{n}^{N} \varepsilon_{n}|n\rangle\langle n|-t \sum_{n}^{N}(|n\rangle\langle n+1|+| n+1\rangle\langle n|)
$$

On-site energies $\varepsilon_{n}$ are randomly distributed within the box $[-W / 2, W / 2]$. All wave functions are localized at $W \neq 0$; incoherent transport can then be modeled by means of the Pauli master equation for the populations $P_{\nu}$ of the quasi-particle states:

$$
\dot{P}_{\nu}=R_{\nu}-\Gamma_{\nu} P_{\nu}+\sum_{\mu}\left(W_{\nu \mu} P_{\mu}-W_{\mu \nu} P_{\nu}\right), \quad W_{\mu \nu}=S\left(\left|\omega_{\mu \nu}\right|\right) G\left(\omega_{\mu \nu}\right) I_{\nu \mu} .
$$

where $R_{\nu}$ is the source term, $\Gamma_{\nu}$ is the total decay rate of state $\nu$ (and describes the drain), $W_{\nu \mu}$ is the scattering rate from the state $|\mu\rangle$ to the state $|\nu\rangle$, which is due to the weak coupling to a phonon system. The rates $W_{\nu \mu}$ are taken to

* Corresponding author: e-mail: a.malyshev@valbuena.fis.ucm.es, Phone: +349139450 12, Fax: +34913944547 
be proportional to the one-phonon spectral density $S(\omega)$ and the probability overlap integral $I_{\nu \mu}=\sum_{n} \Psi_{\mu n}^{2} \Psi_{\nu n}^{2}(\operatorname{see}$ e.g. Refs. [3, 4] and [5] for details), $\omega_{\mu \nu}=E_{\mu}-E_{\nu}$, and $G(\omega)=n(\omega)$ if $\omega>0$ while $G(\omega)=1+n(-\omega)$ if $\omega<0$, with $n(\omega)=[\exp (\omega / T)-1]^{-1}$ being the mean thermal occupation number of a phonon mode with energy $\omega$ (the Boltzmann constant is set to unity while $T$ is the temperature). The model proved to be relevant for J-aggregates [3, 4, 6, 7].

\section{LOCAL LEVEL STATISTICS IN 1D}

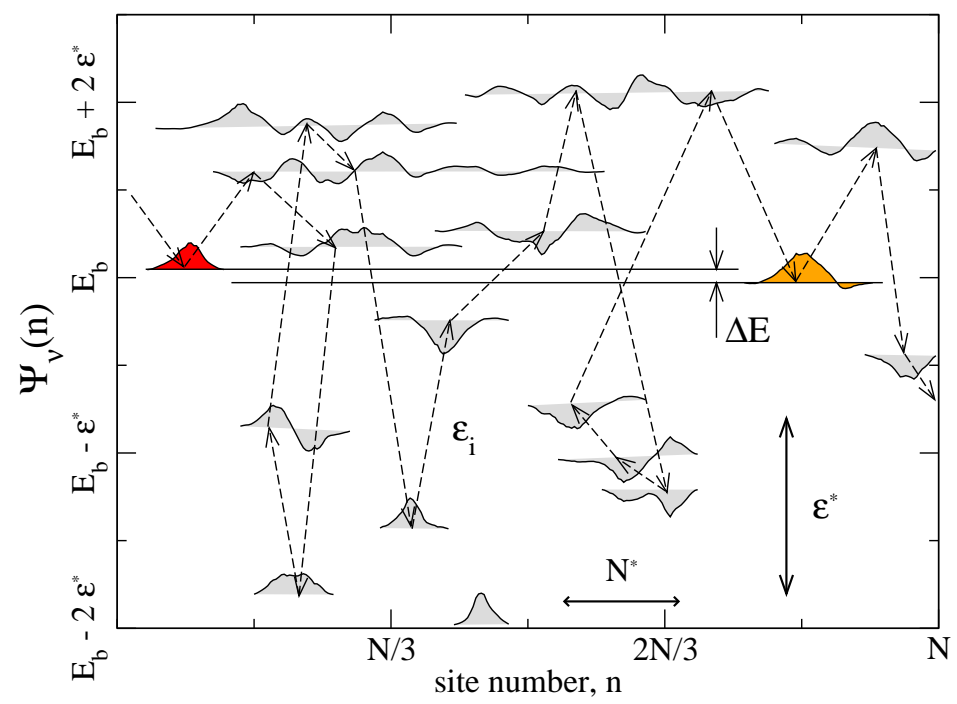

FIG. 1: Wavefunctions and a diffusion trajectory: baselines of each wavefunction represent the energy of the state, wavefunctions are in arbitrary units. Wavefunctions forming the Lifshits tail of the density of states $\left(E_{\nu} \leq E_{b}=-2 t, E_{b}\right.$ is the bare band bottom [at $W=0]$ ) are localized at segments of typical size $N^{*}=N^{*}(W)$. Dashed line - a typical quasi-particle diffusion trajectory: because the hopping probability is proportional to the the overlap integral $I_{\mu \nu}$ the particle hops mostly over well-overlapping states. $\Delta E$ is the smallest energy separation between two distant states, which is relevant for global level statistics $\left(\lim _{N \rightarrow \infty} \Delta E=0\right), \epsilon_{i}$ is the energy spacing for the hop $i$ with the typical spacing $\epsilon^{*} \operatorname{such}$ that $\lim _{N \rightarrow \infty} \epsilon^{*}=$ const.

A typical realization of wavefunctions of the Hamiltonian (1) for a chain of size $N$ and a possible diffusion trajectory is shown in Fig. 1. Note, that the overlap integral $I_{\mu \nu}$ that enters the hopping rate $W_{\mu \nu}$ selects hops between states that overlap well (see also the figure caption for comments). The corresponding energy spacing at hop $i$ between such correlated levels is $\epsilon_{i}$. To account for the correlation we propose the following procedure that is relevant for 1D case: (i) from the full sorted energy set $\left\{E_{\nu}\right\}$ select energies within the window of interest (e.g. ( $\left.-\infty, E_{c}\right]$ where the upper cut-off energy can reasonably be set as $E_{c} \sim E_{b}$ or $E_{c} \sim E_{b}+T, E_{b}$ being the bare band bottom [at $\left.W=0\right]$ ), (ii) sort the selected energy set in the averaged radius-vector for the state $\nu, \bar{x}_{\nu}=\sum_{n}\left|\Psi_{\nu n}\right|^{2} n$ (in $1 \mathrm{D} \bar{x}$ is a number and such sorting is well defined); let $\left\{\bar{E}_{i}: \bar{x}_{i+1} \geq \bar{x}_{i}\right\}$ be the $\bar{x}$-sorted set, then (iii) construct a new energy sequence $\left\{\mathcal{E}_{n}: \mathcal{E}_{n}=\sum_{i}^{n} s_{i}, s_{i}=\left|\bar{E}_{i+1}-\bar{E}_{i}\right|\right\}$, i. e. new energies are cumulative sums of absolute spacings between $\bar{x}$-sorted energies. The set $\left\{\mathcal{E}_{i}\right\}$ takes into account the above-mentioned correlations because sequential states from the set $\left\{\mathcal{E}_{n}\right\}$ are always close in $r$-space, so that overlap integral between them is large. In the next section we calculate the local NLS distribution functions $P_{E_{c}}(S)$ for $\bar{x}$-sorted energy sequences and discuss the results.

\section{NUMERICAL RESULTS AND DISCUSSION}

Fig. 2 demonstrates the local NLS statistics results calculated for $N=4096$ and averaged over 1000 realizations of disorder. Left panel shows the dependence of the mean NLS on the disorder magnitude $W$. In contrast to the global NLS statistics with vanishing mean spacing in the thermodynamic limit, the mean local NLS is independent of the system size. Calculated for $E_{c}=E_{b}$ it determines the characteristic energy scale for the states at the Lifshits tail of the density of states, e.g., the diffusion activation energy at low temperature (the latter was demonstrated for the case of the Frenkel exciton diffusion, see Ref. [3], where the statistics of the hidden energy structure 8 ] was used; such statistics can be viewed as a limiting (less general) case of the proposed local NLS statistics). The characteristic energy $\epsilon^{*}(W)$ of the local NLS for $E_{c}=E_{b}$ is in good agreement with the energy spacing in the hidden energy structure [8]. 
In order to analyze universal correlations between energy levels and compare NLS distribution functions to the standard ones (Poisson and Wigner-Dyson) it is necessary to unfold the raw energy sequence $\left\{\mathcal{E}_{n}\right\}$ (see Ref. $[\underline{9}]$ for details). Right panel of Fig. 2 shows the distribution functions $P_{E_{c}}(S)$ calculated from the unfolded energy sequence for $W=0.3 t$ and different cut-off energies $E_{c}$. Poisson and Wigner-Dyson distributions are shown for comparison. When $E_{c}$ is well below the bare band edge $E_{b}=-2 t$ ( $E_{c}$ is deep in the Lifshits tail) the distribution tends to the Poisson distribution and demonstrates almost no level repulsion. In this case the states are localized far away from each other and there is a vanishing overlap and correlation between them. As the cut-off energy approaches the bare band bottom $E_{b}$ clear sign of level repulsion appears in the local NLS distribution function. However, the distribution does not tend to the Wigner-Dyson one upon increase of $E_{c}$, rather it tends to some limiting distribution. The latter is not surprising because although the $\bar{x}$-sorted sequence is correlated it is a sequence of localized states and no true Wigner-Dyson statistics can be expected. The reason for this is the following: it turns up that there is a non-negligible amount of neighboring segments of the chain with very similar fluctuations of the random potential (averaged over the typical localization volume of the wavefunction, $N^{*}$ ); energies of the states localized at such fluctuations are therefore very close to each other. Such pairs of fluctuations are similar to the system of two identical potential wells or a two-atom molecule; as the result, the "eigenstates" of such pairs of fluctuations remind bonded and anti-bonded states of two-atom molecule and can be very close in energy (subject to the wavefunction overlap). Positions of both states are also very close to each other, so they almost always follow each other in the $\bar{x}$-sorted sequence making even the local NLS statistics less repulsive. Quantitative contribution of such pairs of states to the NLS statistics will be analyzed elsewhere.
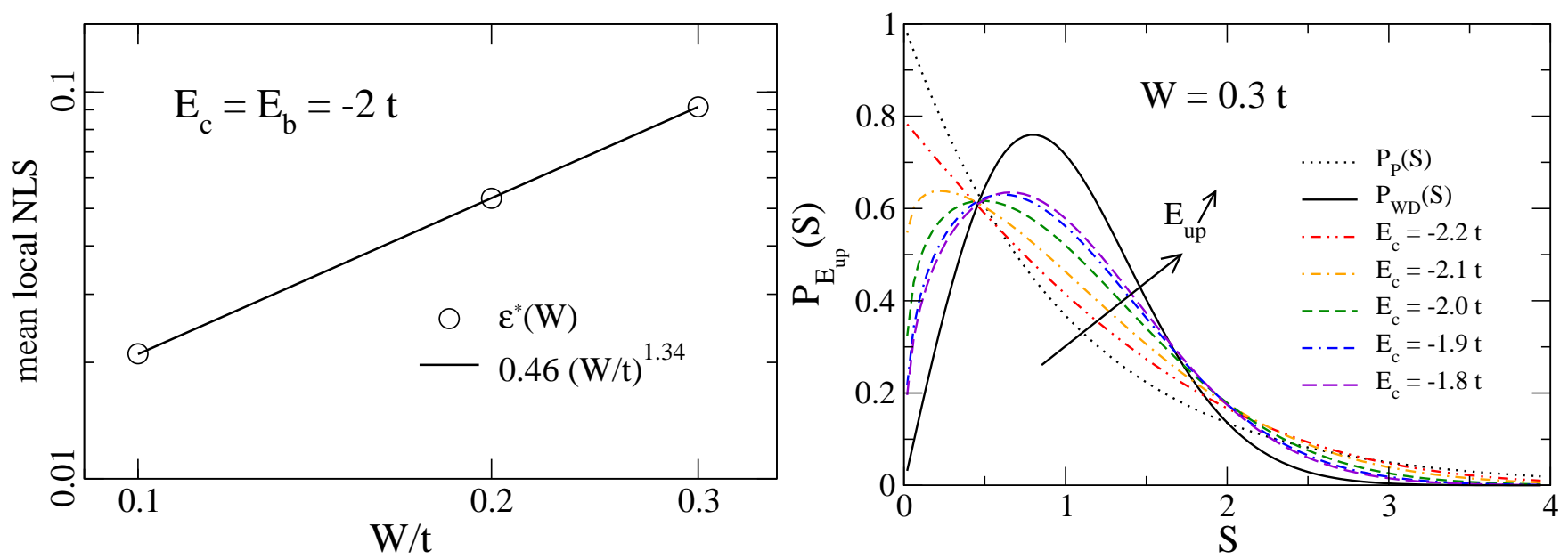

FIG. 2: Left panel - the dependence of the characteristic energy scale $\epsilon^{*}$ on the disorder magnitude $W$ for $E_{c}=E_{b}$. Right panel - local NLS distribution function $P_{E_{c}}(S)$ for the unfolded $\bar{x}$-sorted sequence compared to the Poisson distribution $P_{P}(S)$ (completely uncorrelated levels) and the Wigner-Dyson one $P_{W D}(S)$ (correlated levels).

The dependence of the local NLS statistics on the cut-off energy allows for definition of the diffusion mobility edge in a similar way the conventional mobility edge that separates extended and localized phases is defined. Although no scaling variables or rigorous argumentation is available in the present case (to the best of the author's knowledge), by analogy, we formally define the diffusion mobility edge as the energy $E_{\mu}$ such that:

$$
\left.P_{E_{\mu}}\left(S_{0}\right)\right)-\frac{P_{P}\left(S_{0}\right)+P_{W D}\left(S_{0}\right)}{2}=0
$$

where $P_{P}(S)$ and $P_{W D}(S)$ are Poisson and Wigner-Dyson distributions respectively, and the spacing $S_{0} \sim 1$. In practice, we averaged $E_{\mu}$ over various $S_{0}: 0.1 \leq S_{0} \leq 3$. For all values of disorder magnitude the diffusion mobility edge coincided with the bare band bottom $E_{b}=-2 t$. In order to interpret the later result it is useful to compare the number of states below the mobility edge to the number of the Lifshits tail states $N / N^{*}$ (see Fig. 1), it turns up that for all values of $W$

$$
\int_{-\infty}^{E_{\mu}} \rho(E) d E \approx \frac{N}{N^{*}}, \quad \rho(E)=\left\langle\sum_{\nu}^{N} \delta\left(E-E_{\nu}\right)\right\rangle .
$$

The diffusion mobility edge has therefore a clear physical meaning: it separates the spectral region with states that do not overlap with neighbors (hopping rates between such neighbors are vanishing) from the energy region where 
wavefunctions begin to overlap well: higher (band) states cover one or more tail states and overlap well with them and between each other as well (see Fig. 1). In the latter case hopping rates and diffusion build up drastically. The importance of two-step hops via higher states was discussed in detail in Ref. [3] in connection with the Frenkel exciton diffusion.

\section{CONCLUSIONS}

We have defined local NLS statistics for 1D disordered systems. The local statistics grasps correlation between levels that are involved in hopping transport and is generally more repulsive than the global one. The local NLS distribution in the localized phase changes from the Poisson one to the distribution that demonstrates clear level repulsion, depending on the spectral region and the energy window. Monitoring the change we determine the diffusion mobility edge that has a clear physical meaning: it separates the states with vanishing probability of hopping between neighbors (slow incoherent transport) from the states with large inter-state hopping rate (fast transport regime). Using similar argumentation local level statistics can be defined for higher-dimensional systems for which the common mobility edge can be defined. The mobility edge determined by the local statistics would however be different from the common one because the former is always the diffusion mobility edge. Analysis of higher-dimensional cases is beyond the scope of this paper and will be discussed elsewhere.

AM is grateful to J. Knoester and V. Malyshev for fruitful discussions. Support by INTAS through YSF 03-55-1545 and Spanish Ministry of Education and Science through Ramón y Cajal Program is acknowledged. AM is on leave from Ioffe Institute, St. Petersburg, Russia.

[1] A. D. Mirlin, Phys. Rep. 326, 259 (2000)

[2] Martin Janssen, Phys. Rep. 295, 1 (1998)

[3] A. Malyshev, V. Malyshev, and F. Domínguez-Adame, Chem. Phys. Lett. 371, 417 (2003)

[4] M. Bednarz, V. A. Malyshev, and J. Knoester, Phys. Rev. Lett. 91, 217401 (2003); J. Chem. Phys. 120, 3827 (2004).

[5] M. Shimizu et al, Phys. Rev. B 58, 5032 (1998); M. Shimizu, S. Suto, and T. Goto, J. Chem. Phys. 114, 2775 (2001).

[6] J. Knoester, in Organic Nanostructures: Science and Application (IOS Press, Amsterdam, 2002), pp. 149-186.

[7] D. J. Heijs, V. A. Malyshev, and J. Knoester, Phys. Rev. Lett. 95, 117402 (2005); J. Chem. Phys. 123, 144507 (2005).

[8] V. Malyshev and P. Moreno, Phys. Rev. B 51, 14587 (1995); A. Malyshev and V. Malyshev, Phys. Rev. B 63, 195111 (2001).

[9] M. L. Mehta, Random Matrices, 2nd ed. (Academic Press 1991) 